\title{
Investigating the Role of Ventricular Repolarization Morphology in Surface ECGs for Identifying Patients with a History of Drug-Induced Arrhythmias
}

\author{
JP Couderc, S Kaab, M Hinterseer, S McNitt, X Xia, A Fossa, B Beckmann, S Polonsky, W Zareba \\ University of Rochester, NY, USA; Ludwig-Maximilians University, Munich Germany; \\ Pfizer Global Research development, CT, USA
}

\begin{abstract}
Assessing the propensity of an individual to druginduced arrhythmias when exposed to a QT-prolonging drug is challenging because baseline QT prolongation has limited predictive value. In this study, we investigated the role of computerized ECG parameters quantifying $T$ wave morphology for the identification of individuals who developed drug-induced torsades de pointes (TdPs).

In 34 patients, 5-minute digital ECGs have been acquired at baseline and during sotalol challenge. Seventeen of these patients had a history of drug-induced TdPs. We identified specific baseline features of the Twaves common to patients with history of TdPs. The sotalol-induced changes of $T$-wave morphologies were also different between the two groups suggesting that ECGs may provide relevant information for the identification of patients with an increased propensity to TdPs.
\end{abstract}

\section{Introduction}

The electrophysiological factors contributing to the occurrence of drug-induced TdPs remain unclear. One interesting concept describing the potential triggering factors was described by Hondeghem et al., it emphasises the roles of action potential triangulation, reverse use dependence of the drug and repolarization instability (TRiaD)[1;2]. The changes in action potential shape (triangulation) and the heterogeneity of these potentials across the myocardium was also described as an important arrhythmogenic factor by Antzelevitch[3]: the transmural dispersion of repolarization. Following these concepts, we hypothesize that the patients with a history of drug-induced TdPs may have a certain level of repolarization impairment (heterogeneity, action potential triangulation, reduced repolarization reserve) that may be reflected on the ECG by specific patterns of the T-wave (morphology), as it has been reported in patients with the congenital long QT syndrome[4]. Furthermore, we extend this hypothesis to drug-induced changes of $\mathrm{T}$-wave. The individuals with a history of TdPs could present specific changes of T-wave when they are exposed to torsadogenic drugs such as sotalol.

We have developed a set of ECG markers related to the measurements of the repolarization morphology from digital ECG signals. These markers improved the sensitivity of ECG markers to detect the presence of drugs such as moxifloxacin (Computers in Cardiology 2006)[5].

In this study, we describe the application of these parameters to a novel and independent dataset of ECG signals from individuals with history of TdPs. We aim at strengthening our hypothesis about the important role of T-wave morphology in the risk stratification of patients with a predisposition to drug-induced arrhythmias.

\section{Method}

\subsection{Study population}

The first study group consisted in patients admitted to the University Hospital of Munich (Germany) for documented drug-induced TdPs. They were enrolled in the study after signing informed consent to receive doses of sotalol[6]. The control group consisted in patients who were started on sotalol for prevention of paroxysmal atrial fibrillation and did not experience any TdPs. All individuals were genetically tested for the presence of mutations of the major LQTS genes.

\subsection{Study protocol - ECGs pre-processing}

The study protocol is described in the article published in 2003 by Kaab et al.[7]. Sotalol was given intravenously at a constant rate over a 20 minute interval at a dose of $2 \mathrm{mg} / \mathrm{kg}$ body weight in $50 \mathrm{ml}$ of $0.9 \%$ saline solution in all enrolled patients. The patients with a history of TdPs presented significantly longer sotalol-induced QT prolongation revealing their predisposition to LQTS presented as an impaired repolarization reserve.

\subsection{ECG recordings}


Continuous 5-minute surface 12-lead ECG recordings (Mortara Instrument, Milwaukee, WI, USA) were acquired at rest in supine position at baseline and at 20min steady state phase after injection (corresponding to the time of peak concentration of the drug). The RR intervals and repolarization intervals were based on technology developed at the University of Rochester Medical Center, NY. The COMPAS software provided the location of the end of the T-wave based on a technique identifying the crossing-point between the baseline and the descending slope of the T-wave (leastsquares technique)[8]. The apex of the T-wave relied on a method using a parabola fitting the T-wave where the maximum of the parabola identified the location of the apex.

\subsection{Repolarization parameters}

We report both the scalar fully-computerized and semicomputerized QT interval. The fully-computerized version computes QT in all available beats whereas in the semi-computerized approach the measurements were done in 3 cardiac beats in sinus rhythm from lead V5 (or II) and a trained technician manually adjusted the end of the T-wave if needed. The median value from the three measures was computed.

The vectorcardiographic measurements were based on the singular value decomposition of the repolarization segment defined between the $\mathrm{J}$ point and the point located $220 \mathrm{~ms}$ before the next $\mathrm{R}$ peak when applied to the 12lead ECG signals. This ensures that the analysis encompasses all components of the ventricular repolarization signal and the measurements are independent from the determination of the end of the Twave. The method to measure repolarization characteristics has been described previously[5].

We computed the QT, QTapex and the TpTe intervals (TpTe=QT-QTapex) from the first eigenvector in a fully computerized manner. We used the repolarization signals from the two first eigenvectors to define a set of interval durations depending on the morphology of the T-loop. The duration of these intervals are defined by amplitude thresholds equal to $30 \%$, and $70 \%$ of the maximum vector amplitude of repolarization[5]. The measurements are called early repolarization duration (ERD) and late repolarization duration (LRD). These measurements are illustrated in Figure 1.

\section{Results}

One of the patients had atrial fibrillation during the ECG recording. This ECG was removed from the analysis resulting in a group of 16 patients with a history of TdPs and 17 individuals free of such history.
Table 1: ECG Baseline Characteristics

\begin{tabular}{lcc}
\hline & $(-)$ TdPs & $(+)$ TdPs \\
RR & $925 \pm 162$ & $922 \pm 142$ \\
PR & $161 \pm 28$ & $153 \pm 27$ \\
QRS & $97 \pm 15$ & $95 \pm 11$ \\
QTc Semi- & $425 \pm 36$ & $450 \pm 39^{*}$ \\
computerized & $422 \pm 37$ & $450 \pm 39^{*}$ \\
QTc computerized & $340 \pm 24$ & $347 \pm 35$ \\
QTc apex & $435 \pm 32$ & $461 \pm 45^{*}$ \\
QTc & $108 \pm 35$ & $108 \pm 31$ \\
TpTe & $35 \pm 8$ & $44 \pm 13^{* *}$ \\
ERD30\% & $57 \pm 13$ & $71 \pm 22^{* *}$ \\
ERD50\% & $28 \pm 5$ & $35 \pm 17$ \\
LRD30\% & $43 \pm 5$ & $55 \pm 32$ \\
LRD50\% & \\
\hline
\end{tabular}

$*=<0.06, * * \mathrm{p}<0.03(-)$ TdPs: patients without a history of TdPs, (+) TdPs: patients with a history of TdPs. All measurements are expressed in milliseconds.

The Table 1 provides the average values and standard deviation for most investigated parameters for the baseline values. The Table 2 reports changes between baseline and sotalol for the two groups: individuals with and without history of TdPs.

Table 2: Sotalol-induced changes repolarization morphology

\begin{tabular}{|c|c|c|c|}
\hline & (-) TdPs & (+)TdPs & $P$ values \\
\hline RR & $175 \pm 98 *$ & $201 \pm 101 *$ & 0.34 \\
\hline PR & $3 \pm 27$ & $5 \pm 22$ & 0.83 \\
\hline QRS & $0 \pm 9$ & $3 \pm 13$ & 0.49 \\
\hline QTc $\ddagger$ & $80 \pm 48^{*}$ & $101 \pm 45^{*}$ & 0.22 \\
\hline QTc & $74 \pm 42 *$ & $82 \pm 54 *$ & 0.54 \\
\hline QTc apex & $54(37) \pm 24 *$ & $75(53) \pm 37^{*}$ & 0.06 \\
\hline QTc & $55(61) \pm 27^{*}$ & $94(103) \pm 46^{*}$ & 0.01 \\
\hline TpTe & $6(7) \pm 20$ & $36(25) \pm 41^{*}$ & 0.01 \\
\hline LRD50\% & $7 \pm 10^{*}$ & $21 \pm 27^{*}$ & 0.07 \\
\hline LRD70\% & $8(9) \pm 13$ & $27(28) \pm 30 *$ & 0.03 \\
\hline
\end{tabular}

All repolarization measurements were heart-rate corrected based on linear pooled-formula. The RR 
intervals are significantly longer after sotalol (+TdPs: $201 \pm 101 \mathrm{msec}$ and - TdPs: $175 \pm 98 \mathrm{msec}, \mathrm{p}<0.05)$, but the bradycardiac effect of the drug is not different between groups $(\mathrm{p}=0.34)$.

Figure 1.Location of the ERD and LRD intervals on the T-loop and on the first eigenvector (lower panel).
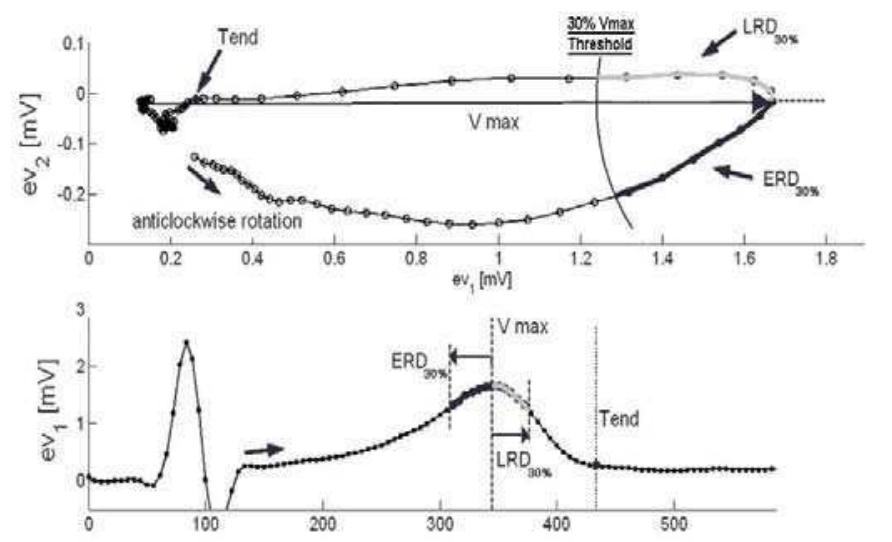

Our analysis reveals that sotalol significantly prolongs the TpTe interval only in the group with a history of TdPs (36 \pm 41 vs. $6 \pm 20 \mathrm{msec}, \mathrm{p}=0.01)$. The measurements of the early and late part of the repolarization interval (ERD and LRD) confirm the observations based on QT, QT apex and TpTe intervals. The sotalol-induced delay of the repolarization is increased in both groups but only the late part of the repolarization process (LRD70\%) is significantly longer in the ECGs of individuals with a history of TdPs $(27 \pm 30$ vs. $8 \pm 13 \mathrm{msec}, \mathrm{p}=0.03)$.

On average, the QT interval measurement is $25 \mathrm{msec}$ longer in the $(+) \mathrm{TdPs}$ group $(\mathrm{p}=0.06)$. According to the vectorial parameters, this prolongation is localized within the early part of the repolarization segment as shown by ERD30\% and ERD50\%. ERD30\% is $9 \mathrm{msec}$ longer in the $+\mathrm{TdPs}$ group $(\mathrm{p}=0.02)$ and this prolongation reaches 14 msec with ERD50\% ( $\mathrm{p}=0.03)$. Interestingly, this delay in the early phase of the repolarization segment is not captured by the QT apex interval revealing that the morphology of the T-wave primarily drives the measurement of this delay.

Binary logistic regressions were implemented in order to find which of the baseline information could help predict the presence of a history of TdPs. QTc, QTc apex, TpTe, ERDx\% and LRDx\% were included in the design. Based on both stepwise and best subsets, the model revealed that $\mathrm{ERD}_{30 \%}$ was the strongest predictor of a history of TdPs. For each incremental $1 \mathrm{msec}$ duration of $\mathrm{ERD}_{30 \%}$, there was $8.4 \%$ increased probability of having a history of TdPs $(p=0.036)$. When selecting a model with two parameters, QTc entered the model with a negligible contribution and a low level of statistical significance $(\mathrm{p}=0.12)$.

\section{Discussion and conclusions}

We report the analysis of repolarization morphology in two groups of individuals with and without a history of drug-induced TdPs. First, our analysis reveals a longer QT interval in baseline ECGs of patients with a history of TdPs. This prolongation was prominently located in the early part of the T-wave prior to its apex. Second, the drug sotalol induced significant prolongation of the QTc interval that was larger in the group of patients with a history of TdPs, more importantly this increased delay of repolarization was located in the latest part of the $\mathrm{T}$-wave (TpTe interval and $\mathrm{LRD}_{\mathrm{x} \%}$ ).

Our study evidenced that at baseline, a large set of patients with a history of drug-induced TdPs has a specific repolarization profile similar to the one we observed in ECGs of healthy subjects with moxifloxacin namely changes in morphology of the $\mathrm{T}$-wave prior to the T-wave apex and associated with a delayed process[5;9]. And, sotalol affects both the early and late parts of the Twave with significant prolongations of these intervals as shown in Table 2.

Several clinical investigations have reported gene-specific patterns of the T-wave morphologies in patients with the congenital LQTS suggesting a direct link between specific ion-kinetics abnormalities and changes of the morphology of the T-wave[10-15]. Under the assumption that there is a triangulation of the action potential according to the TRIaD concept, the presence of an individual predisposition to TdPs could be associated with specific T-wave patterns. Because the triangulation affects mainly the phase 2 of the AP, one might expect that the early phase of the T-wave might be the most affected as we observed using our $\mathrm{ERD}_{\mathrm{x} \%}$ indices.

The interval between the T-wave apex and the T-wave end may reflect the global transmural voltage dispersion demonstrated to be an important component of the genesis of TdPs in an experimental model[16]. Our results suggest that the late part of the T-wave, measured either by the TpTe interval or the $\mathrm{LRD}_{\mathrm{x}} \%$ parameters, is more significantly prolonged in patients with history of TdPs than the individuals without arrhythmia.

\section{Acknowledgements}

We would like to thank Dr. Meijian Zhou from iCardiac Technologies Inc., and Dr. Craig Trost from Pfizer Inc. for their valuable support during the analysis of this data. Part of this research was financed by iCardiac Technologies Inc. and Pfizer Global Research and Development. 


\section{References}

[1] Hondeghem LM, Carlsson L, Duker G. Instability and triangulation of the action potential predict serious proarrhythmia, but action potential duration prolongation is antiarrhythmic. Circulation 2001 Apr 17;103(15):2004-13.

[2] Hondeghem LM. TRIad: foundation for proarrhythmia (triangulation, reverse use dependence and instability). Novartis Found Symp 2005;266:235-44.

[3] Belardinelli L, Antzelevitch C, Vos MA. Assessing predictors of drug-induced torsade de pointes. Trends Pharmacol Sci 2003 Dec;24(12):619-25.

[4] Moss AJ, Zareba W, Benhorin J, Locati EH, Hall WJ, Robinson JL, et al. ECG T-wave patterns in genetically distinct forms of the hereditary long QT syndrome [see comments]. Circulation 1995 Nov 15;92(10):2929-34.

[5] Couderc JP, Vaglio M, Xia X, McNitt S, Hyrien O. Electrocardiographic Method for Identifying Drug-induced Repolarization Abnormalities Associated with a Reduction of the Rapidly Activating Delayed Rectifier Potassium Current. IEEE Engineering in Medicine and Biology Society, New-York 2006 p. 4010-5.

[6] Kaab S, Hinterseer M, Nabauer M, Steinbeck G. Sotalol testing unmasks altered repolarization in patients with suspected acquired long-QT-syndrome--a case-control pilot study using i.v. sotalol. Eur Heart J 2003 Apr;24(7):64957.

[7] Kaab S, Hinterseer M, Nabauer M, Steinbeck G. Sotalol testing unmasks altered repolarization in patients with suspected acquired long-QT-syndrome--a case-control pilot study using i.v. sotalol. Eur Heart J 2003 Apr;24(7):64957.

[8] Lepeschkin E, Surawicz B. The measurement of the Q-T interval of the electrocardiogram. Circulation 1952 Sep;6(3):378-88

[9] Couderc J, Vaglio M, McNitt S, Hyrien O, Zareba W, Moss A.J. Electrocardiographic method for identifying moxifloxacin-induced ventricular repolarization abnormalities. Computers in Cardiology; Valencia Spain: Computers in Cardiology; 2006 p. 705-8.

[10] Couderc JP, McNitt S, Hyrien O, Vaglio M, Xia J, Polonsky S, et al. Electrocardiographic Abnormalities of Repolarization Induced by Moxifloxacin: Improving the Detection of Subtle $\mathrm{I}_{\mathrm{Kr}}$-Inhibition. Drug Safety J. In press 2007.

[11] Couderc JP, McNitt S, Xia J, Zareba W, Moss AJ. Repolarization morphology in adult LQT2 carriers with borderline prolonged QTc interval. Heart Rhythm 2006 Dec;3(12):1460-6.

[12] Malfatto G, Beria G, Sala S, Bonazzi O, Schwartz PJ. Quantitative analysis of $\mathrm{T}$ wave abnormalities and their prognostic implications in the idiopathic long QT syndrome. J Am Coll Cardiol 1994 Feb;23(2):296-301.

[13] Padrini R, Butrous G, Statters D, Camm AJ, Malik M. Morphological algebraic models of the TU-wave patterns/in idiopathic long QT syndrome. Int $\mathbf{J}$ Cardiol 2001 Feb;77(2-3):151-62.

[14] Priori SG, Napolitano C, Diehl L, Schwartz PJ. Dispersion of the QT interval. A marker of therapeutic efficacy in the idiopathic long QT syndrome. Circulation 1994 Apr;89(4):1681-9.

[15] Viitasalo M, Oikarinen L, Swan H, Glatter KA, Vaananen
$\mathrm{H}$, Fodstad $\mathrm{H}$, et al. Ratio of late to early T-wave peak amplitude in 24-h electrocardiographic recordings as indicator of symptom history in patients with long-QT Syndrome types 1 and 2. J Am Coll Cardiol 2006 Jan 3;47(1):112-20.

[16] Antzelevitch C, Burashnikov A, Di Diego JM. Cellular and Ionic Mechanisms underlying Arrhythmogenesis. In: Gussak I, Antzelevitch C, editors. Cardiac Repolarization: Bridging Basic and Clinical Sciences.Totowa: Humana Press; 2004. p. 201-51.

Address for correspondence.

Jean-Philippe Couderc

601 Elmwood Ave, Box 546

Rochester, NY, USA

Tel: 585-275-1096

Fax: 585-2735283

Jean-philippe.couderc@heart.rochester.edu 